\title{
Ictal time-irreversible intracranial EEG signals as markers of the epileptogenic zone
}

Kaspar Schindler ${ }^{1 *}$, Christian Rummel ${ }^{2}$, Ralph Andrzejak ${ }^{3}$, Marc Goodfellow ${ }^{4,5,6}$, Frédéric Zubler $^{1}$, Eugenio Abela ${ }^{2}$, Roland Wiest ${ }^{2}$, Claudio Pollo ${ }^{7}$, Andreas Steimer ${ }^{1+}$, Heidemarie Gast $^{1+}$

${ }^{1}$ Sleep-Wake-Epilepsy-Center, Department of Neurology, Inselspital, Bern University Hospital, University Bern, Switzerland

${ }^{2}$ Support Center for Advanced Neuroimaging (SCAN), Institute of Diagnostic and Interventional Neuroradiology, University Hospital, Inselspital, University of Bern, Switzerland

${ }^{3}$ Department of Information and Communication Technologies, Universitat Pompeu Fabra, Barcelona, Spain

${ }^{4}$ College of Engineering, Mathematics and Physical Sciences, University of Exeter, Exeter, UK ${ }^{5}$ Centre for Biomedical Modelling and Analysis, University of Exeter, Exeter, UK ${ }^{6}$ EPSRC Centre for Predictive Modelling in Healthcare, University of Exeter, Exeter, UK ${ }^{7}$ Department of Neurosurgery, Inselspital, Bern University Hospital, University Bern, Switzerland

${ }^{+}$Contributed equally and share last authorship.

\section{* Corresponding author:}

Kaspar Schindler, MD, PhD

Managing Director

Sleep-Wake-Epilepsy-Center

Department of Neurology

Inselspital, Bern University Hospital

Switzerland

Tel.: +41 (0)31 6324547

Fax: +41(0)316329679

E-mail: kaspar.schindler@insel.ch 
Keywords: seizure dynamics - quantitative EEG - symbolic analysis - pre-surgical evaluation - complex networks.

\begin{abstract}
Objective: To show that time-irreversible EEG signals recorded with intracranial electrodes during seizures can serve as markers of the epileptogenic zone.

Methods: We use the recently developed method of mapping time series into directed horizontal graphs (dHVG). Each node of the $\mathrm{dHVG}$ represents a time point in the original intracranial EEG (iEEG) signal. Statistically significant differences between the distributions of the nodes' number of input and output connections are used to detect time-irreversible iEEG signals.
\end{abstract}

Results: In 31 of 32 seizure recordings we found time-irreversible iEEG signals. The maximally time-irreversible signals always occurred during seizures, with highest probability in the middle of the first seizure half. These signals spanned a large range of frequencies and amplitudes but were all characterized by saw-tooth like shaped components. Brain regions removed from patients who became post-surgically seizure-free generated significantly larger time-irreversibilities than regions removed from patients who still had seizures after surgery. Conclusions: Our results corroborate that ictal time-irreversible iEEG signals can indeed serve as markers of the epileptogenic zone and can be efficiently detected and quantified in a time-resolved manner by dHVG based methods.

Significance: Ictal time-irreversible EEG signals can help to improve pre-surgical evaluation in patients suffering from pharmaco-resistant epilepsies. 


\section{Introduction}

The aim of pre-surgical evaluation of patients suffering from pharmacoresistant epilepsies is to delineate the epileptogenic zone, which has been defined as the minimal area of cortex that must be resected to produce seizure-freedom (Luders et al., 2006). Since there is still no unique diagnostic method to precisely, directly and unequivocally identify the epileptogenic zone, it is crucial to extract as much information as possible from existing modalities. Intracranial EEG (iEEG) is currently the technology with the highest spatiotemporal resolution and the best signal-to-noise ratio to record the electric brain activity during human epileptic seizures. In recent years, modern methods of quantitative signal analysis have progressively proved to yield diagnostically and prognostically important information for planning epilepsy surgery (Andrzejak et al., 2015). These methods can therefore complement classic visual EEG analysis by an expert electroencephalographer since they provide more objective information. Quantitative analysis methods often differ in terms of the particular features of a signal that they extract. An important feature of electrographic signals in the epileptic brain is temporal asymmetry. This is observed, for example, in signals with uneven slopes of their rising and falling parts (or "phases" to use the vocabulary of visual EEG interpretation). In the language of quantitative signal analysis, one refers to this feature as time-irreversibility, which means that statistical properties are different depending on whether a signal is analysed forward or backward in time (Daw et al., 2000). The importance of this asymmetry as a characteristic of epileptiform signals has long been known to epileptologists, even being illustrated almost forty years ago by Gloor in a seminal book chapter (see Figure 1 in Gloor's publication) about the use of EEG in the differential diagnosis of epilepsy (Gloor, 1977). Importantly, time-irreversibility excludes linear Gaussian processes as generators of a signal (Weiss, 1975) and therefore may be used as an efficient test for the presence of deviations from this assumption. Such deviations have been 
demonstrated to be characteristic of seizure generating brain regions (Andrzejak et al., 2001a, Andrzejak et al., 2001b). Although temporally asymmetric "skew shaped" iEEG signals may be detected by an experienced electroencephalographer, it is difficult to visually assess the amount of "temporal skewness". Therefore, quantitative methods are needed that are robust and efficient when applied to real-world time series such as ictal iEEG recordings, which are always contaminated by observational noise and are typically analysed using short moving windows to minimize their non-stationarity. A recently developed method that is based on socalled directed Horizontal Visibility Graphs (dHVG) has been demonstrated to have many of the required characteristics (Lacasa et al., 2008, Lacasa et al., 2012) and has already been shown to detect time-irreversibility in a set of EEG signals that had been recorded extracranially with skin surface electrodes and intracranially with hippocampal depth electrodes and during interictal and ictal states (Donges et al., 2013). The strongest timeirreversibilities were found in iEEG recorded during seizures (Donges et al., 2013). However, it is not yet known to what extent time-irreversible iEEG signals are indeed markers of the epileptogenic zone in patients suffering from different types of pharmacoresistant epilepsies. In the present study we retrospectively quantify time-irreversible iEEG signals in a dataset from patients with known postsurgical outcome. We find that time-irreversibility is maximal in data recorded during seizures, and that it is predominantly largest for saw-tooth like signals. Furthermore, patients who became post-surgically seizure-free had brain regions removed that generated iEEG signals with significantly stronger time-irreversibility than patients who still had seizures. We therefore demonstrate the usefulness of timeirreversibility analysis to aid delineation of the epileptogenic zone.

\section{Methods}

\subsection{Patients}


We included 16 patients of the epilepsy surgery program of the Inselspital Bern in this study (11 female, 5 male; median age 31.0 y, IQR 15 y, range 19-59 y). 12 patients had temporal lobe epilepsy (7 with normal MR findings), 2 had frontal lobe and 2 parietal lobe epilepsy. Demographic data about this patient collective has recently been published (Rummel et al., 2015). Inclusion criteria were the availability of at least one year of post-surgical follow up (median $3.0 \mathrm{y}$, IQR $1.3 \mathrm{y}$ ) and that high resolution pre- and post-operative T1-weighted MRI had been registered. Fusion of MRIs with CT-images of implanted intracranial electrodes then allowed precisely identifying the brain regions from which the electrodes had recorded. The decision for intracranial EEG diagnostics, the specific electrode implantation scheme and the decision on surgery were solely based on clinical assessment and were made prior to and independently from the present retrospective study.

We confirm that we have read the Journals position on issues involved in ethical publication and affirm that this report is consistent with those guidelines. The study has been approved by the Ethics Committee of the Kanton of Bern (Id No: 2016-00257 (041/16)).

\section{2 iEEG Recordings}

iEEG signals were recorded intracranially by strip, grid or depth electrodes (AD-TECH, Wisconsin, USA), using a NicoletOne ${ }^{\mathrm{TM}}$ recording system with a C64 amplifier (VIASYS Healthcare Inc., Madison, Wisconsin, USA). The reference electrode was placed extracranially between 10-20 positions Fz and Cz. Depending on whether more or less than 64 iEEG signals were recorded the sampling rate was 512 or $1024 \mathrm{~Hz}$, respectively. iEEG signals recorded with $1024 \mathrm{~Hz}$ were down-sampled to $512 \mathrm{~Hz}$ prior to our quantitative analysis. All iEEG signals were re-referenced against the median of all the channels free of permanent artifacts as judged by visual inspection by two experienced electroencephalographers (K.S. and H.G.). In addition to anti-aliasing filtering, mandatory for 
proper sampling and down-sampling, iEEG signals were digitally band-pass filtered between 0.5 and $150 \mathrm{~Hz}$ using a fourth-order Butterworth filter. Forward and backward filtering was applied to minimize phase distortions. All the pre-processing was done using Matlab (MathWorks, Natick, Massachusetts, USA).

\subsection{Visual iEEG analysis}

Seizure onsets were visually identified by two experienced and board-certified electroencephalographers (K.S. and H.G.) as the time of the earliest ictal iEEG change following a systematic approach (see for example (Litt et al., 2001)). We first identified unequivocal ictal signals and then moved backwards in time to the instance when the first sustained change of iEEG relative to the ongoing background signals occurred. Repetitive high amplitude spike-and-wave signals or flattening due to, or followed by, low voltage fast activity were the most frequently observed changes. Unequivocal seizure onset was defined as any epileptiform signal pattern that became clearly identifiable without knowing that a seizure followed. Seizure termination was determined as the time point when epileptiform signals vanished, which often manifested itself as a generalized and sudden decrease of amplitude and frequency (Schindler et al., 2007, Afra et al., 2015).

\subsection{Quantitative iEEG analysis: Horizontal Visibility Graphs and time-irreversibility}

The recently developed method of Horizontal Visibility Graphs (HVG) consists of mapping a time series such as an iEEG signal into a network (Lacasa et al., 2008, Luque et al., 2009). The rationale for turning an ordered set of values - i.e. the time series - into a different mathematical object is that this allows invoking other analysis methods and assessing the data from an alternative perspective (Nunez et al., 2012). HVG are particularly interesting for the analysis of iEEG signals because they do not require the setting of parameters and they can 
be implemented efficiently. Fast computation is particularly relevant when analysing large data sets such as (long-term) multi-channel iEEG time series. For our study we use directed HVG (dHVG) that are sensitive to temporal (a)symmetries (Lacasa et al., 2012). In Figure 1, the construction of a dHVG is illustrated for a short, artificially generated time series. This

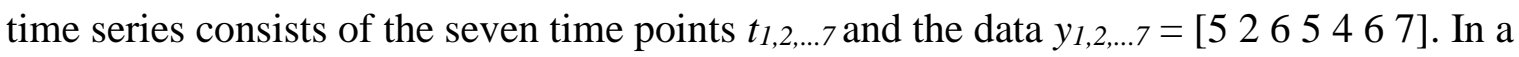
HVG each time point of the original time series corresponds to a node. Two nodes $i$ and $j$ are connected if there is no data point in between them that is higher than $y_{i}$ or $y_{j}$ (Luque et al., 2009). Put in formal terms, the nodes $i$ and $j$ are connected if the following geometrical - socalled convexity - condition is fulfilled within the time series:

$$
\min \left(y_{i}, y_{j}\right)>y_{n} \text { for all } i<n<j
$$

This construction algorithm yields a graph, in which the nodes are connected by undirected links. By considering the natural flow of time from the past to the future it is easy to introduce directed connections between the nodes. The binary connection matrix for the directed Horizontal Visibility Graph (dHVG) derived from the artificial time series is displayed in Figure 1B. The entry at row $i$ and column $j$ is set to 1 if a directed link from $i$ to $j$ exists, otherwise it remains 0 . Since by construction links point towards the future, the connection matrix contains positive entries in its upper right triangular part only. Another characteristic of the connection matrix is that the so-called "superdiagonal", i.e. the diagonal directly above the main diagonal at $i=j$, is filled with ones. This reflects that all nodes are connected with their successors - simply because there are no intermediate data points. The nodes of the dHVG can then be characterised by their input degree $k_{i n}$, which is the sum of the incoming links and their output degree $k_{\text {out }}$ Input and output degrees are efficiently computed by taking the sum along each column or along each row of the connection matrix, 
respectively. The process of assigning an integer (input or output) degree to each time point of the original time series is an elegant and efficient symbolization process (Daw et al., 2003), in which the number of symbols does not need to be (more or less arbitrarily) specified a-priori but naturally emerges from the structure of the time series (Lacasa et al., 2015).

In Figure 2A a real-world iEEG signal of $1 \mathrm{~s}$ duration and sampled at $512 \mathrm{~Hz}$ is displayed. The connection matrix of its corresponding $\mathrm{dHVG}$ is shown in Figure 2B. As in the case of the short, artificially generated signal shown in Figure 1, the typical characteristics of a dHVG are clearly seen. All positive entries are again found in the right upper triangular part, and the superdiagonal is filled with ones. The input and output degrees are displayed as bar plots and in Figure 2C their frequency distributions are plotted. Donges et al. (2013) have recently shown that time-irreversibility can be quantified by using standard statistical tests for the equality of the empirical frequency distributions of input- and output degrees. In the case shown in Figure 2, a two-sample Kolmogorov Smirnov test (KS test) yields $p=0.67$ and thus one cannot reject the hypothesis that the input and the output degrees are drawn from the same distribution. In the framework of dHVG this result implies that the iEEG signal is timereversible. In other words, the degree distributions are not significantly different when the iEEG signal is analysed forwards or backwards in time, which therefore allows a quantification of temporal asymmetry. Although theoretically the KS test is based on the assumption of independent samples - which is not the case for node degrees of a HVG - its utility for testing time (ir)reversibility has recently been demonstrated empirically (Donges et al., 2013). Notice that the input degree of the original iEEG signal is the output degree of the signal assessed backwards. To quantify the strength of irreversibility we use $I$, defined as the negative logarithm with base 10 of the $p$ value yielded by the two-sample Kolmogorov 
Smirnov test of the hypothesis that $k_{\text {in }}$ and $k_{\text {out }}$ of the dHVG are drawn from the same distribution.

$$
I=-\log 10(p)
$$

dHVG is a nonparametric and robust analysis method. For example, iEEG signals may be monotonically distorted by differences in local electrical impedance, but any scaling by a positive and non-decreasing monotonic function will leave the ranks and thus the corresponding dHVG unchanged. An important further advantage of dHVG compared to different methods such as ordinal patterns (Bandt et al., 2002, Schindler et al., 2012) is that except for the duration of the moving analysis window - no additional parameters have to be set.

\subsection{Time-resolved analysis and correction for multiple comparisons}

Each analysed iEEG contained $30 \mathrm{~s}$ before the visually identified seizure onset, the seizure, and $30 \mathrm{~s}$ after seizure termination. Recordings were analysed channel-wise and in a timeresolved manner by using a moving window of one second duration $T=512$ sampling points (sp), which was advanced forward in time without overlap in steps of one second (512 sp). For each analysis window the iEEG signal was mapped onto the corresponding $\mathrm{dHVG}$ and time irreversibility was quantified by $I$ (equation 2 , above). We corrected the statistical significance level of $p=0.01$ by the number of tests computed, which amounts to the number of non-overlapping analysis windows times the number of iEEG signals. In other words, we applied a conservative Bonferroni correction.

Like for pre-processing of the iEEG data, quantitative analysis was performed using Matlab (MathWorks, Natick, Massachusetts, USA). 


\section{Results}

We analysed the iEEG recordings of the first two seizures of all 16 patients (median number of seizures per patient: 4.5, IQR 5.5, range 2-14). We did not analyse all seizures to prevent biasing our results towards those patients who had recordings from a higher number of seizures, and two seizures was the minimal number of seizures recorded for every patient. We found statistically significant time-irreversible iEEG signals in 31 of the 32 recordings (97\%). In Figure 3 the iEEG and analysis results of patient \#4 who suffered from temporal lobe epilepsy and became seizure-free after surgery is shown as a representative case. Figure 3A displays the iEEG signals including $30 \mathrm{~s}$ before and $30 \mathrm{~s}$ after the seizure, with the corresponding dynamics of $I$ shown as a color-coded plot in Figure 3B. The dotted-lined crosshairs indicate the global maximal time-irreversibility, i.e. the iEEG channel $s_{\text {Max }}$ that recorded the maximally time-irreversible signal at time $t_{\text {Max }}$. In this case $s_{\text {Max }}$ coincided with the channel, where the first ictal iEEG changes occurred. Note that there are also strong time irreversibilities in channels 13 and 32. The spatio-anatomical relationships between these three channels are illustrated in Figure 4, where post-implantation CT scan and pre-operative MRI were fused as previously described (Rummel et al., 2015).

In relative seizure times (i.e. time relative to seizure onset divided by seizure duration), $t_{\text {Max }}$ for all 31 recordings with significant time-irreversibility was $0.45 \pm 0.3$ (mean \pm standard deviation) (range: 0-0.98). In Figure 5 the maximally time-irreversible signal emerging at $t_{\text {Max }}$ $=44 \mathrm{~s}$ in channel $s_{\text {Max }}=38$ is displayed together with the connection matrix of the corresponding $\mathrm{dHVG}$ and the probability distributions of the input- and output degrees. Visual inspection of the iEEG signal (Figure 5A) reveals a signal with approximately 7 periods within the moving window of $1 \mathrm{~s}$ duration and an evident temporal asymmetry due to its saw-tooth shape with increasing segments that are much slower compared to the 
decreasing segments. Clearly this signal looks different when observed from the past to the future (left to right) than from the future to the past (right to left). This strong temporal asymmetry is reflected in the input and output degrees of the dHVG and the highly significant difference yielding $I_{\max }=69$.

Figure 6 presents a synopsis of all 31 maximally time-irreversible signals, with signals sorted according to the strength of their time-irreversibilities. While these signals contain a large range of frequencies and amplitudes, all of them display some saw-tooth shaped components, which become more easily visible when inspecting the normalized signals depicted in the right panel.

Figure 7A demonstrates that the maximally time-irreversible iEEG signals always occurred during the seizures and were observed with highest probability in relative seizure time period $1 / 6<t<2 / 6$, i.e. in the middle of the first seizure half as measured in relative seizure time. If time-irreversible signals are markers of epileptogenicity as proposed by previous studies (Donges et al., 2013) then there should be a positive association between the strength of timeirreversibility generated by the brain regions that were removed and post-surgical seizure freedom. We indeed found that $I_{\text {Max }}$ was significantly larger in the iEEG signals generated by the brain regions removed in post-surgically seizure-free patients (Figure 7B) compared to non-seizure-free patients.

\section{Discussion}

Here we have shown that time-irreversible iEEG signals occur during most seizures and are markers of the epileptogenic zone. Specifically, using a recently developed rank-based and parameter free method, which implicitly contains a data-driven symbolization step, we have demonstrated that iEEG signal time-irreversibility reveals complex dynamics, which may be exploited to gain diagnostically relevant information. Our results are consistent with the 
interpretation that surgical resection should aim at removing those brain regions that generate the iEEG signals with strongest temporal asymmetry (i.e. greatest time irreversibility). These results are important because they may improve pre-surgical evaluation by increasing the amount of diagnostically relevant information gained from iEEG recordings. They underline that not only classic, linear signal characteristics such as amplitude and frequency should be considered, because other, complementary and quantifiable aspects such as the shape of the signals can be highly relevant (Andrzejak et al., 2001b, Andrzejak et al., 2012).

Our results are in line with and thus corroborate the work of several other groups. Our results confirm the findings of Donges et al. (Donges et al., 2013), but they also support older results of van der Heyden et al. (van der Heyden et al., 1996, van der Heyden et al., 1999) who used a delay-vector based method to differentiate ictal from non-ictal iEEG signals based on their time-irreversibility.

Since time-irreversibility is not present in time series generated by linear Gaussian processes (Weiss, 1975), our results may be put in the broader context of characterizing epileptogenic brain areas by quantifying a departure from the assumption of linearity in the electrical signals they generate. In this regard our results are in line with a series of previous studies that reported nonlinearities in epileptogenic brain regions (Pijn et al., 1997, Andrzejak et al., 2001a, Andrzejak et al., 2001b, Andrzejak et al., 2012). In a recent study we analysed the same iEEG data set with several linear, nonlinear, uni- and multivariate methods and found that nonlinear interdependence is a good predictor for postsurgical outcome (Rummel et al., 2015). However, the dHVG based method modified by Donges et al. (Donges et al., 2013) and originally developed by Lacasa et al. (Lacasa et al., 2008, Luque et al., 2009) is computationally more efficient and arguably conceptually simpler than the surrogate based approaches used in our previous study. Furthermore, time-(ir)reversibility is a signal characteristic that has also been known to be an important cue for visual analysis (Gloor, 
1977). As a simplifying rule, iEEG signals that increase and decrease with similar slope, i.e. which are time-reversible, are often not pathologic - though the converse does not apply as illustrated by the physiologically occurring "saw-tooth-wave" of REM sleep. Thus not only the amplitudes and dominant period of an iEEG signal are relevant, but also its overall shape. However, while temporal asymmetry may be noticed by visual inspection, it is difficult to quantify. Our results show that the dHVG based approach applied in the present study is a robust and computationally efficient tool to measure temporal asymmetry and therefore complement and guide visual iEEG interpretation.

From a technical point of view, one might wonder if using a moving analysis window of $1 \mathrm{~s}$ duration and high-pass filtering the iEEG signals at $0.5 \mathrm{~Hz}$ might introduce a certain bias towards temporal asymmetry, because the slowest signals only partially fit into the analysis window. Therefore we re-computed the analysis after high-pass filtering the iEEG signals at $2 \mathrm{~Hz}$ and we found almost identical results (data not shown). Importantly, still all the iEEG signals selected according to $I_{\text {Max }}$ displayed the saw-tooth like shaped components and the group statistic shown in Figure 6 remained unchanged at $p=0.002$.

Interestingly, the ictal iEEG signals with maximal time-irreversibility (Figure 6) are qualitatively similar to the signals we previously identified as having maximal signal redundancy (Schindler et al., 2012). In that study we used ordinal pattern analysis in a smaller group of patients without assessing the relationship between surgically removed brain regions and post-operative seizure freedom. Most signals with maximal time-irreversibility are relatively slow and therefore much smaller sampling rates could be used compared to, for example, high frequency oscillations as markers of epileptogenicity (Modur et al., 2011, Jacobs et al., 2012).

Considering that many different processes contribute with unknown relative proportions to the generation of iEEG signals (Buzsáki et al., 2012) the neurophysiologic basis of the 
observed time-irreversibility has to remain speculative. Though unspecific in terms of mechanisms an intuitive interpretation could be that temporal asymmetry reflects a dysbalance of synchronization and desynchronization and/or excitation and inhibition (Dehghani et al., 2016) in the underlying cellular networks (McCormick et al., 2001).

Intriguingly, time-irreversibility may also be observed on much larger time scales that include the seizure as a whole. Comparing the pre-ictal and the immediate postictal time periods in regard to iEEG characteristics or neuropsychiatric state of the patient (Ito, 2010) clearly reveals that the dynamics leading into a seizure are qualitatively different from those occurring at seizure termination (Afra et al., 2015) and bringing about the post-ictal state. If pre-seizure dynamics were similar to the often slowly fading, post-ictal phenomena, seizure prediction might be much easier to achieve (Mormann et al., 2007).

There are several interesting ways to extend our present study. One important question of immediate practical interest is the spatial relationship between the temporally asymmetric signals we found as markers of the epileptogenic zone and other signal characteristics such as ictal (Modur et al., 2011, Malinowska et al., 2015) and interictal fast oscillations (Cho et al., 2014, Kerber et al., 2014, Okanishi et al., 2014, Gliske et al., 2016) . Clarifying this relationship will be relevant for assessing how much additional information can be provided by quantifying the time irreversibility of iEEG signals. A related issue is the search for time irreversible signals during the interictal time period; in particular during different sleep stages.

Another line of investigation will be to use HVG node degrees (and other network measures) not only to characterize single iEEG signals but to also measure the dynamic interdependences between several iEEG signals. One elegant way to implement such an approach was recently demonstrated by Lacasa et al. (Lacasa et al., 2015) who converted 
financial time series into HVG and then constructed a multilayer ("multiplex") network, in which each layer consisted of one HVG. By studying the mutual information between the degree distributions of these layers they were able to assess the interdependences between the original financial time series.

A limitation of our study is the relatively small number of patients and therefore we should seek to replicate our findings in a larger cohort that includes a greater diversity of types of epilepsy.

Finally, time-irreversible iEEG signals may aid recent efforts to build Bayesian statistical based generative models of multi-channel iEEG (Dauwels et al., 2011, Steimer et al., 2015). Such models may be used to simulate the effect of modulating subsets of iEEG channels. Due to the very large number of ways in which subgroups of channels may be chosen, preselection based on specific and objective signal characteristics such as time-irreversibility could considerably reduce the computational load.

\section{Conclusions}

We conclude firstly that removing brain areas that generate time-irreversible iEEG signals during seizures is associated with post-surgical seizure freedom. Secondly, time-irreversible iEEG signals are characterized by an asymmetry of increases and decreases and may occur throughout the seizure. And finally, directed horizontal visibility graphs (dHVG) are a computationally highly efficient and robust method to quantify time-irreversibility of iEEG signals with high temporal resolution.

\section{Conflict of interest}

None of the authors have potential conflicts of interest to be disclosed. 


\section{Acknowledgments}

K.S. gratefully acknowledges support by the Swiss National Science Foundation (SNF 32003B_155950). H.G. gratefully acknowledges support by a Research Grant of the Inselspital Bern. R.G.A. acknowledges funding from the Volkswagen foundation and was supported by the Spanish Ministry of Economy and Competitiveness (Grant FIS2014-54177R). This project has received funding from the European Union's Horizon 2020 research and innovation programme under the Marie Sklodowska-Curie grant agreement No 642563 (R.G.A.). MG gratefully acknowledges the financial support of the EPSRC via grant EP/N014391/1, funding from Epilepsy Research UK via grant number A1007 and was generously supported by a Wellcome Trust Institutional Strategic Support Award (WT105618MA). 


\section{References}

Afra P, Jouny CC, Bergey GK. Termination patterns of complex partial seizures: An intracranial EEG study. Seizure. 2015;32:9-15.

Andrzejak RG, David O, Gnatkovsky V, Wendling F, Bartolomei F, Francione S, et al. Localization of Epileptogenic Zone on Pre-surgical Intracranial EEG Recordings: Toward a Validation of Quantitative Signal Analysis Approaches. Brain Topogr. 2015;28:832-7. Andrzejak RG, Lehnertz K, Mormann F, Rieke C, David P, Elger CE. Indications of nonlinear deterministic and finite-dimensional structures in time series of brain electrical activity: dependence on recording region and brain state. Phys Rev E 2001a;64:061907. Andrzejak RG, Schindler K, Rummel C. Nonrandomness, nonlinear dependence, and nonstationarity of electroencephalographic recordings from epilepsy patients. Phys Rev E 2012;86:046206.

Andrzejak RG, Widman G, Lehnertz K, Rieke C, David P, Elger CE. The epileptic process as nonlinear deterministic dynamics in a stochastic environment: an evaluation on mesial temporal lobe epilepsy. Epilepsy Res. 2001b;44:129-40.

Bandt C, Pompe B. Permutation Entropy: A Natural Complexity Measure for Time Series. Phys Rev Lett. 2002;88:174102.

Buzsáki G, Anastassiou CA, Koch C. The origin of extracellular fields and currents - EEG, ECoG, LFP and spikes. Nat Rev Neurosci. 2012;13:407-20.

Cho JR, Koo DL, Joo EY, Seo DW, Hong SC, Jiruska P, et al. Resection of individually identified high-rate high-frequency oscillations region is associated with favorable outcome in neocortical epilepsy. Epilepsia. 2014;55:1872-83.

Dauwels J, Yu H, Wang X, Vialette FB, Latchoumane C-FV, Jeong J, et al. Inferring Brain Networks through Graphical Models with Hidden Variables. In: Langs G, Rish I, Grosse- 
Wentrup M, Murphy B, editors. Neural Information Processing Systems. Berlin: Springer; 2011. p. 194-201.

Daw CS, Finney CEA, Kennel MB. Symbolic approach for measuring temporal “irreversibility”. Phys Rev E 2000;62:1912-21.

Daw CS, Finney CEA, Tracy ER. A review of symbolic analysis of experimental data. Rev Sci Instrum. 2003;74:915-30.

Dehghani N, Peyrache A, Telenczuk B, Le Van Quyen M, Halgren E, Cash SS, et al. Dynamic Balance of Excitation and Inhibition in Human and Monkey Neocortex. Sci Rep. $2016 ; 6: 23176$.

Donges FD, Donner RV, Kurths J. Testing time series irreversibility using complex network methods. EPL. 2013;102:10004.

Gliske SV, Irwin ZT, Davis KA, Sahaya K, Chestek C, Stacey WC. Universal automated high frequency oscillation detector for real-time, long term EEG. Clin Neurophysiol. 2016;127:1057-66.

Gloor P. The EEG and differential diagnosis of epilepsy. In: Van Duyn H, Donker DNJ, Van Huffelen AC, editors. Current Concepts in Clinical Neurophysiology. The Hague: NV Drukker Trio; 1977. p. 9-21.

Ito M. Neuropsychiatric evaluations of postictal behavioral changes. Epilepsy Behav. 2010;19:134-7.

Jacobs J, Staba R, Asano E, Otsubo H, Wu JY, Zijlmans M, et al. High-frequency oscillations (HFOs) in clinical epilepsy. Prog Neurobiol. 2012;98:302-15.

Kerber K, Dumpelmann M, Schelter B, Le Van P, Korinthenberg R, Schulze-Bonhage A, et al. Differentiation of specific ripple patterns helps to identify epileptogenic areas for surgical procedures. Clin Neurophysiol. 2014;125:1339-45. 
Lacasa L, Luque B, Ballesteros F, Luque J, Nuno JC. From time series to complex networks: the visibility graph. Proc Natl Acad Sci U S A. 2008;105:4972-5.

Lacasa L, Nicosia V, Latora V. Network structure of multivariate time series. Sci Rep. 2015;5:15508.

Lacasa L, Nuñez A, Roldán É, Parrondo JMR, Luque B. Time series irreversibility: a visibility graph approach. EPJ B. 2012;85:217.

Litt B, Esteller R, Echauz J, D'Alessandro M, Shor R, Henry T, et al. Epileptic seizures may begin hours in advance of clinical onset: a report of five patients. Neuron. 2001;30:51-64. Luders HO, Najm I, Nair D, Widdess-Walsh P, Bingman W. The epileptogenic zone: general principles. Epileptic Disord. 2006;8 Suppl 2:S1-9.

Luque B, Lacasa L, Ballesteros F, Luque J. Horizontal visibility graphs: exact results for random time series. Phys Rev E 2009;80:046103.

Malinowska U, Bergey GK, Harezlak J, Jouny CC. Identification of seizure onset zone and preictal state based on characteristics of high frequency oscillations. Clin Neurophysiol. 2015;126:1505-13.

McCormick DA, Contreras D. On the cellular and network bases of epileptic seizures. Annu Rev Physiol. 2001;63:815-46.

Modur PN, Zhang S, Vitaz TW. Ictal high-frequency oscillations in neocortical epilepsy: implications for seizure localization and surgical resection. Epilepsia. 2011;52:1792-801. Mormann F, Andrzejak RG, Elger CE, Lehnertz K. Seizure prediction: the long and winding road. Brain. 2007;130:314-33.

Nunez AM, Lacasa L, Gomez JP, Luque B. Visibility Algorithms: A Short Review. In: Zhang Y, editor. New Frontiers in Graph Theory: INTECH; 2012. p. 119-52. 
Okanishi T, Akiyama T, Tanaka S, Mayo E, Mitsutake A, Boelman C, et al. Interictal high frequency oscillations correlating with seizure outcome in patients with widespread epileptic networks in tuberous sclerosis complex. Epilepsia. 2014;55:1602-10.

Pijn JP, Velis DN, van der Heyden MJ, DeGoede J, van Veelen CW, Lopes da Silva FH. Nonlinear dynamics of epileptic seizures on basis of intracranial EEG recordings. Brain Topogr. 1997;9:249-70.

Rummel C, Abela E, Andrzejak RG, Hauf M, Pollo C, Muller M, et al. Resected Brain Tissue, Seizure Onset Zone and Quantitative EEG Measures: Towards Prediction of PostSurgical Seizure Control. PLoS One. 2015;10:e0141023.

Schindler K, Gast H, Goodfellow M, Rummel C. On seeing the trees and the forest: singlesignal and multisignal analysis of periictal intracranial EEG. Epilepsia. 2012;53:1658-68. Schindler K, Leung H, Elger CE, Lehnertz K. Assessing seizure dynamics by analysing the correlation structure of multichannel intracranial EEG. Brain. 2007;130:65-77.

Steimer A, Zubler F, Schindler K. Chow-Liu trees are sufficient predictive models for reproducing key features of functional networks of periictal EEG time-series. Neuroimage. 2015;118:520-37.

van der Heyden MJ, Diks C, Pijn JP, Velis DN. Time reversibility of intracranial human EEG recordings in mesial temporal lobe epilepsy. Phys Lett A. 1996;216:283-8.

van der Heyden MJ, Velis DN, Hoekstra BP, Pijn JP, van Emde Boas W, van Veelen CW, et al. Non-linear analysis of intracranial human EEG in temporal lobe epilepsy. Clin Neurophysiol. 1999;110:1726-40.

Weiss G. Time-Reversibility of Linear Stochastic Processes. J Appl Probab. 1975;12:831-6. 


\section{Figure Legends}

Figure 1. Mapping a time series into a directed Horizontal Visibility Graph (dHVG). A. An artificially generated short time series consisting of 7 datapoints $y_{i=1: 7}=\left[\begin{array}{llllll}5 & 2 & 6 & 5 & 4 & 6\end{array}\right]$ occurring at time points $t_{i=1: 7}$. Each time point is represented by a node in the $\mathrm{dHVG}$, and two nodes $i$ and $j$ are connected if there is no intermediate datapoint larger or equal to $\min \left(y_{i}, y_{j}\right)$. Whether this condition is fulfilled can be graphically illustrated by drawing a horizontal line from $\min \left(y_{i}, y_{j}\right)$ to time $t_{j}$ (or $t_{i}$ ) and testing for intersection by any $y_{n}$ for $i<n<j$. For example $t_{1}$ and $t_{3}$ are connected, because $y_{2}=2$ does not intersect the horizontal line drawn from $y_{1}$ (which is $\left.\min \left(y_{1}, y_{3}\right)\right)$ to $t_{3}$. This connection is represented by the arrow, which points by definition from the past towards the future. As another example, consider $t_{5}$ and $t_{7}$, which are not connected because $y_{6}$ intersects the dotted line drawn from $y_{5}$ to $t_{7}$.

B. The connection (or ,adjacency“) matrix of the dHVG derived from the time series shown in A. This binary matrix contains non-zero entries in the right upper triangle only, reflecting that by construction the connections are exclusively directed from the past to the future following the natural temporal order. The sums along each column are the input degrees $k_{\text {in }}$ of each node and are displayed as the darker blue bar graph above the connection matrix. The sums along each row are the output degrees $k_{\text {out }}$ of each node and are shown by the brighter blue bars on the right of the connection matrix. Assigning an integer input and output degree to each time point is an efficient symbolisation procedure, in which the number of symbols emerges from the intrinsic structure of the time series, rather than being arbitrarily prespecified.

Figure 2. Quantifying time-(ir)reversibility of an iEEG signal. A. iEEG signal of $1 \mathrm{~s}$ duration, sampled at $512 \mathrm{~Hz}(\mathrm{sp}=$ sampling points). B. Binary connection matrix of the dHVG derived from the iEEG signal shown in A. All connections are found in the upper right 
triangle, reflecting that by construction the connections are directed from past to future time points and not vice versa. The sums along the columns shown in the darker blue bar plot above the connection matrix represent the input degrees $k_{\text {in }}$ of the nodes. The sums along each row are displayed as brighter blue bars on the right and correspond to the output degrees $k_{\text {out }}$ The probability distributions of the input and the output degrees are shown in C. For this signal the hypothesis that the distribution of $k_{\text {in }}$ is different from the distribution of $k_{\text {out }}$ cannot be rejected because the two-sample Kolmogorov Smirnov test yields $p=0.67$. Thus the relatively symmetrically increasing and decreasing iEEG signal shown in $\mathbf{A}$ is not timeirreversible as assessed by the input and output degree distributions of its dHVG, which are statistically indistinguishable.

Figure 3. Dynamics of time-irreversibility of iEEG signals. A. Peri-ictal iEEG recorded from the second seizure of patient \#4 who had structural temporal lobe epilepsy due to hippocampal sclerosis and became seizure free after seletive amygdala-hippocampectomy. iEEG seizure onset as defined by visual analysis occurs at time $=0 \mathrm{~s}$. B. Time resolved irreversibility analysis as quantified by $I=-\log 10(p)$, where $p$ results from a two-sample Kolmogorov Smirnov test between the input and output degrees of the dHVG derived from a moving window of duration $\mathrm{T}=1 \mathrm{~s}$ (512 sampling points). $I$ has been Bonferroni corrected for multiple comparisons and only results with $p<0.01 /(42 \times 319=13398)=7.4 \times 10^{-7}$ are displayed. Those iEEG signals are labelled with numbers that were recorded from brain areas, which were subsequently surgically removed. The crosshair (orange dotted lines) marks the iEEG channel $s_{\text {Max }}=38$ and the time point $t_{\text {Max }}=44 \mathrm{~s}$ when the maximally time irreversible signal occurred. In this case the first ictal iEEG changes were also recorded in iEEG channel 38, i.e. coinciding with $s_{\text {Max }}$. The spatio-anatomical relationships between iEEG channels 38, 32 and 13 in which the strongest time-irreversibilities were recorded are 
shown in Figure 4 and the high-resolution iEEG signal $s_{\text {Max }}$, horizontal visibility graph and degree distributions are displayed in Figure 5.

Figure 4. The spatio-anatomical relationships of the three iEEG channels 38,32 and 13, where the strongest time-irreversibilities were recorded. In $s_{\operatorname{Max}}=38$ also the first ictal iEEG changes occurred and thus this region corresponds to the "core" of the seizure onset zone. Strong time-irreversibilities were almost simultanously recorded in channels 38 and 13 (which corresponds to the tip of the depth electrode inserted from temporo-laterally) and later in channels 32, which is positionned over the lateral temporo-polar region (compare Figure 3B).

Figure 5. The maximally time-irreversible iEEG signal recorded during the second seizure of patient \#4 in iEEG channel $s_{\text {Max }}=38$ and at $t_{\text {Max }}=44 \mathrm{~s}$ (see. Figure 3B). A. The iEEG signal has a very obvious, temporally asymmetric ,saw-tooth“-like shape with relatively slow increasing and much faster decreasing parts. The signal repeats itself approximately 7 times within one second. B. Binary connection matrix of the corresponding dHVG. The input degrees $k_{\text {in }}$ and output degrees $k_{\text {out }}$ are shown in the darker and lighter blue bar graphs, respectively, following the scheme of Figures 1 and 2 . These bar plots clearly demonstrate a large difference between $k_{\text {in }}$ and $k_{\text {out }}$. This impression is quantitatively confirmed by the frequency distributions of $k_{\text {in }}$ and $k_{\text {out }}$ displayed in $\mathbf{C}$, which are highly significantly different ( $\mathrm{p}=6.26^{*} 10^{-70}$ from a Kolmogorov-Smirnov two-sample test) yielding $I=-69$ (see Figure 5).

Figure 6. Synopsis of all 31 maximally time-irreversible iEEG signals recorded. \#Pat is the number of the patient with the asterisk $(*)$ indicating post-surgical seizure freedom. sMax denotes the iEEG channel, in which the maximally time-irreversible signal occurred. IMax 
quantifies time-irreversibility (see equation 2 in method section). The iEEG signals are sorted from top to bottom by decreasing time-irreversibility, i.e. decreasing $I_{\text {Max }}$. Crosses $\left(^{+}\right)$ indicate that the brain region from which the signal was recorded was surgically removed. In contrast to the left column, where signals are plotted on the original scale, they are depicted using the same scale in the right column to ease comparison. There, signals are normalized by subtracting the mean from each signal and dividing by its standard deviation. After normalization the saw-tooth like signal components become more clearly visible even in signals with relatively small amplitudes. $\mathrm{sd}=$ standard deviation.

Figure 7. A. Histogram of $t_{M a x}$ as measured in relative seizure time, i.e. time relative to seizure onset divided by duration of seizure shows that all of the maximally time-irreversible iEEG signals occurred during the seizures. They were most frequently recorded in the second of the six seizure time periods of equal duration, but they may occur during the whole seizure. "preSz" and "postSz" denote the 30 s pre- and post-seizure time periods. B. Association between surgical resection of brain regions that generate maximally timeirreversible signals and postsurgical seizure freedom. $I_{\text {Max }}$ in the iEEG signal recorded from surgically removed brain regions was significantly larger in postsurgically seizure-free patients ( $p=0.002$, two-sided Wilcoxon rank sum test). The horizontal black lines represent the medians of the distributions. 


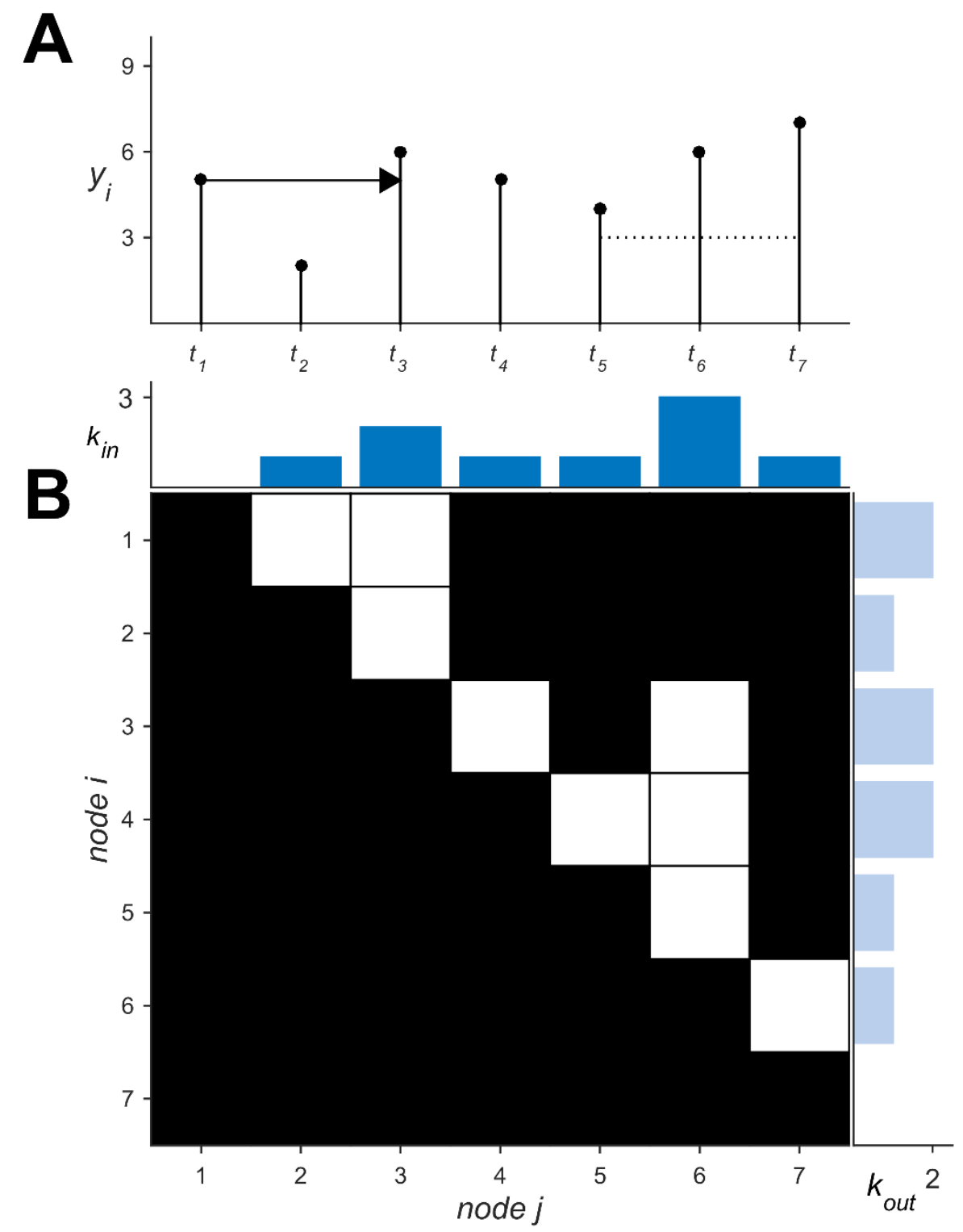


A
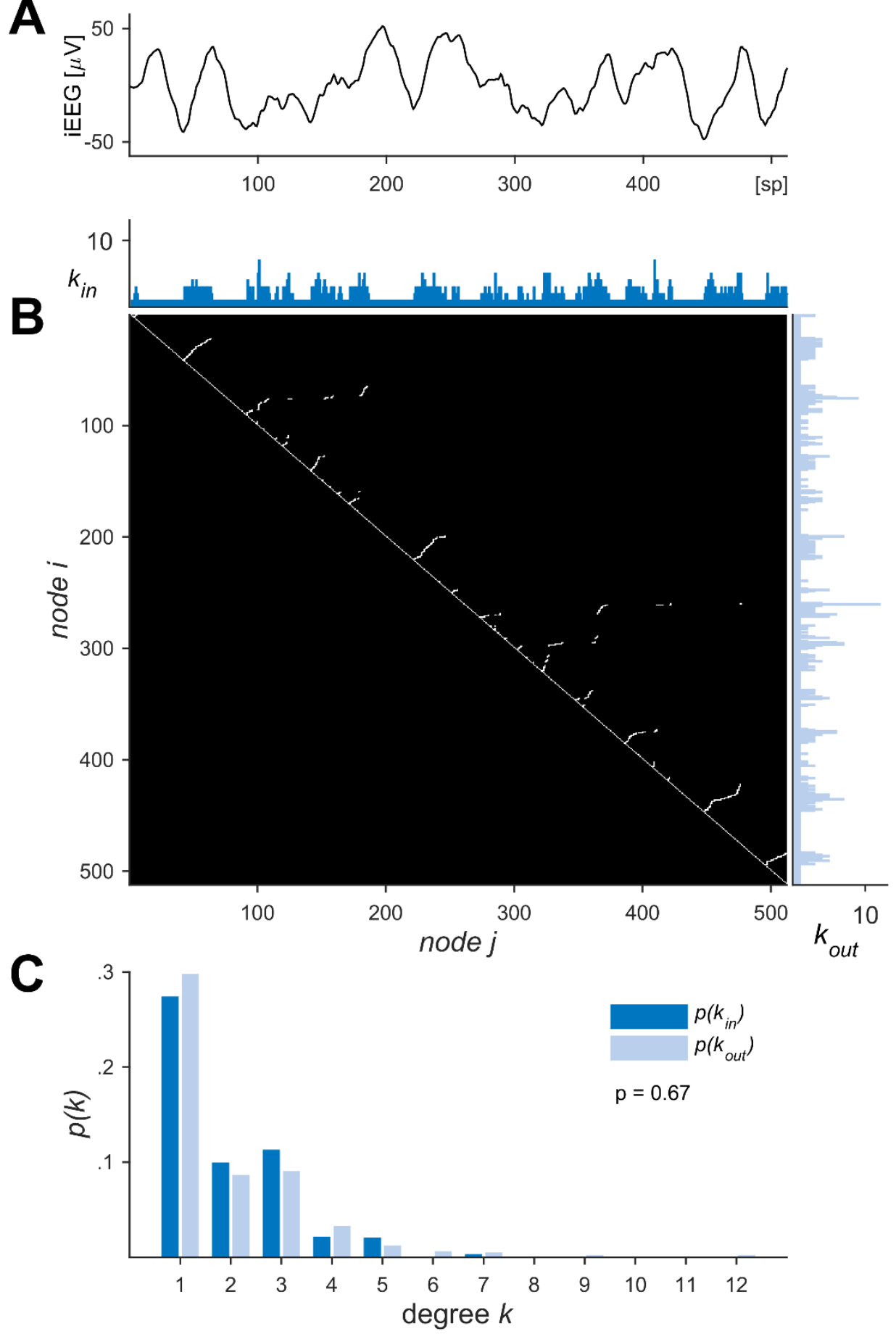







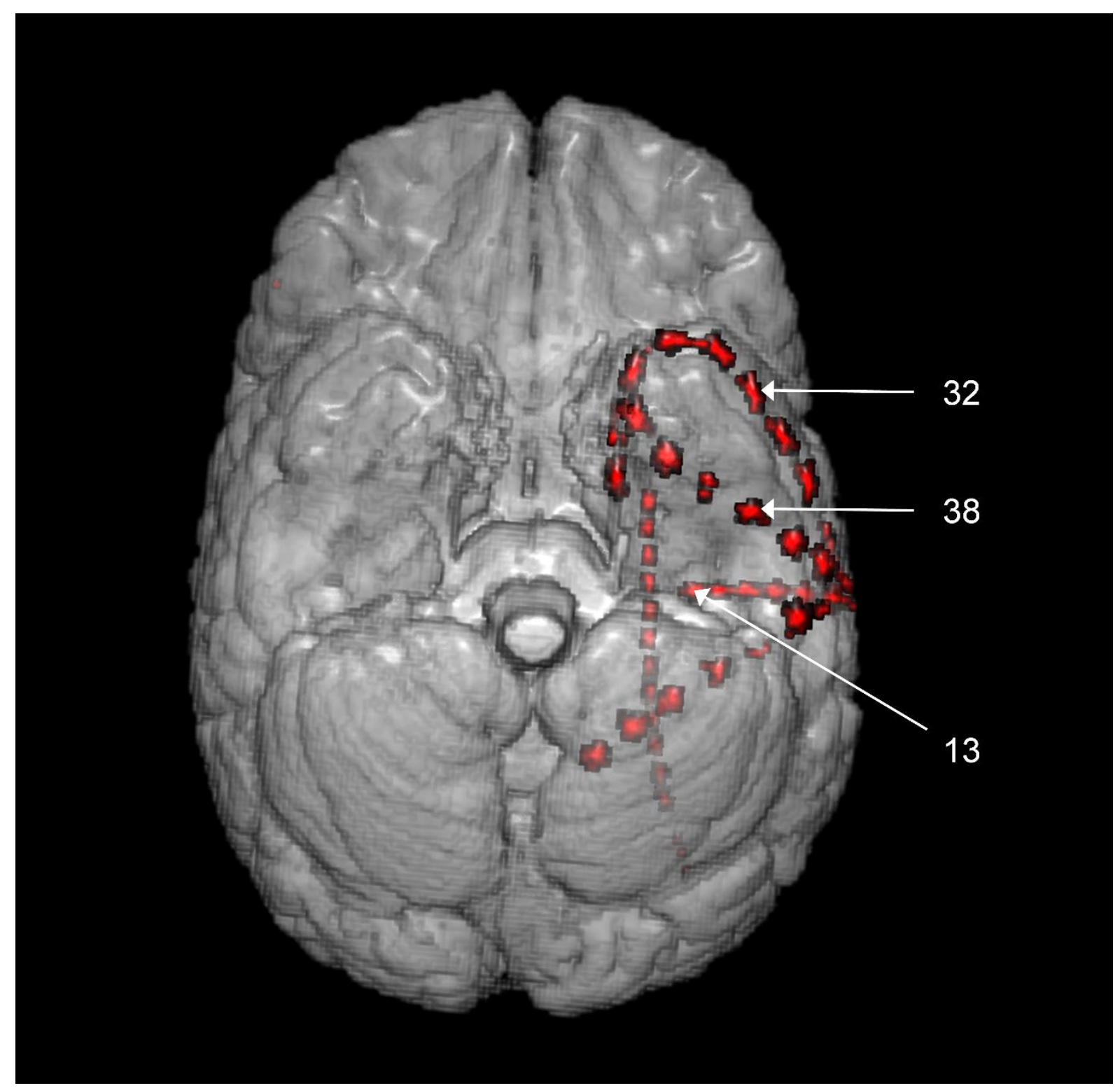


A
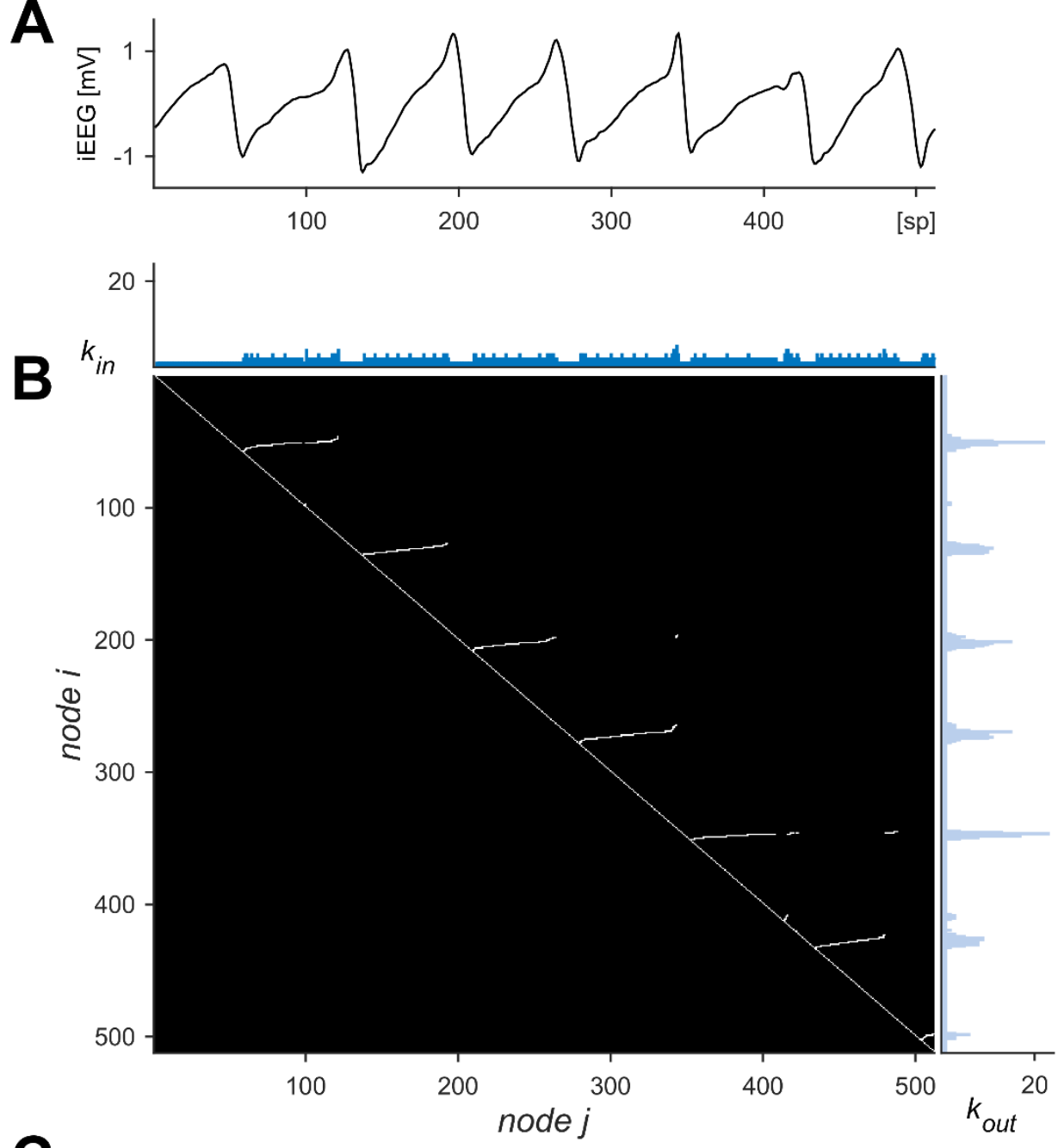

C

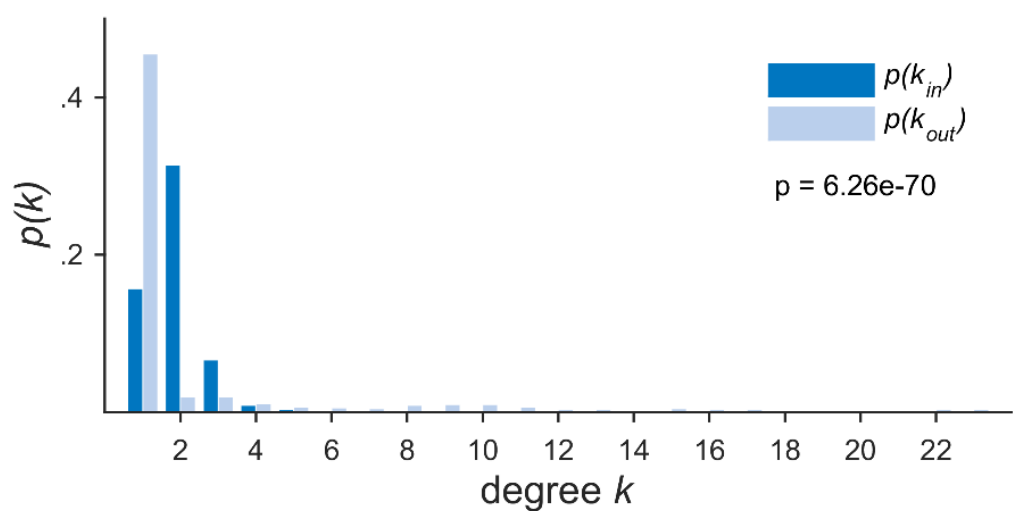




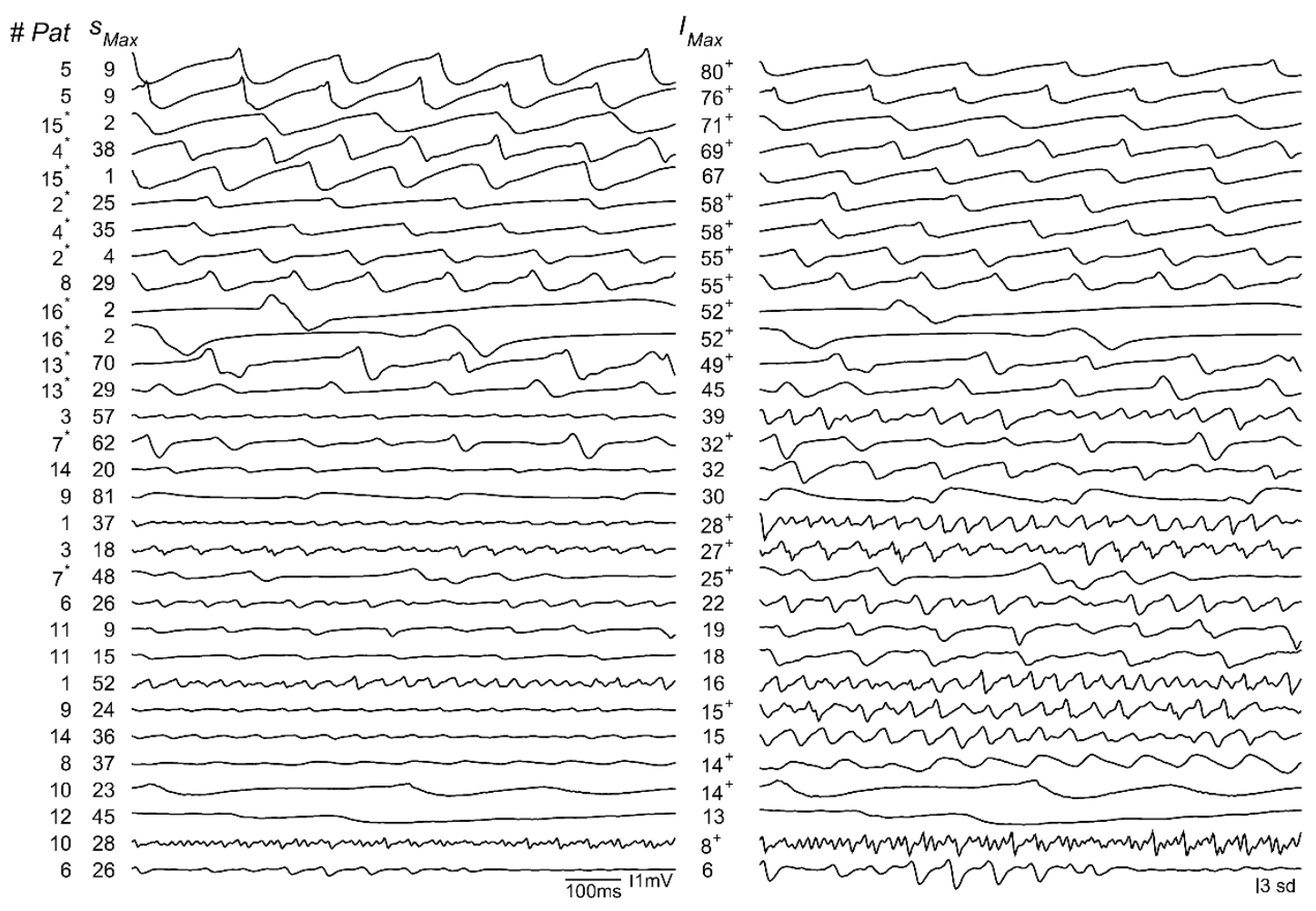




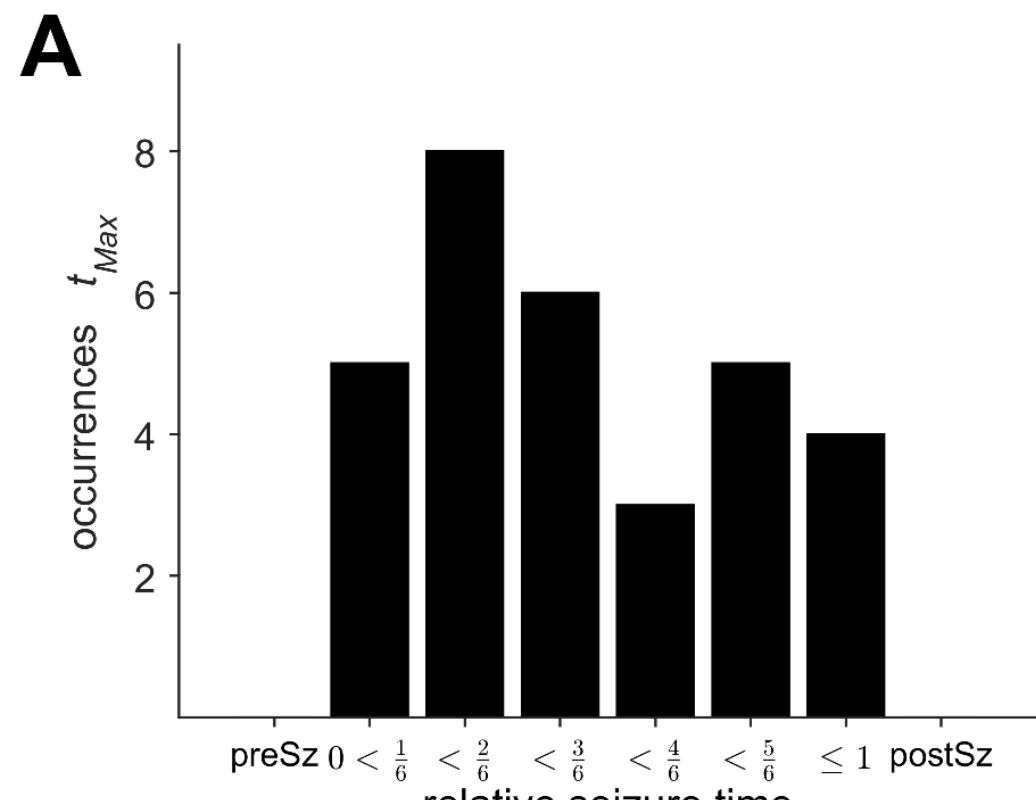

relative seizure time

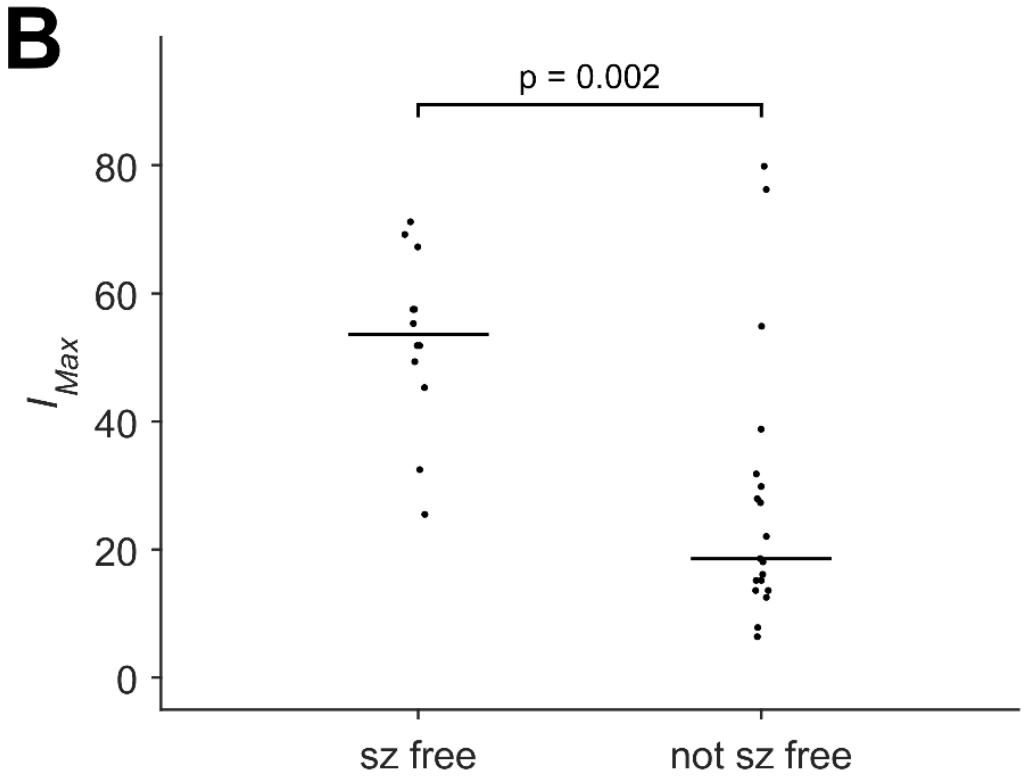

